\title{
Qualidade de carnes provenientes de cortes da carcaça de cordeiros e de ovinos adultos
}

\author{
Rafael Silvio Bonilha Pinheiro ${ }^{1}$, Américo Garcia da Silva Sobrinho², Hirasilva Borba Alves \\ de Souza ${ }^{2}$, Sandra Mari Yamamoto ${ }^{3}$
}

\footnotetext{
${ }^{1}$ Doutorando em Zootecnia (Produção Animal) da Universidade Estadual Paulista, Faculdade de Medicina Veterinária e Zootecnia, Campus de Botucatu, SP, Brasil.

2 Universidade Estadual Paulista, Faculdade de Ciências Agrárias e Veterinárias, Campus de Jaboticabal, SP, Brasil.

3 Pós-doutoranda do Programa de Pós-graduação em Zootecnia da Universidade Estadual de Maringá.
}

\begin{abstract}
RESUMO - Com o objetivo de estudar as características qualitativas da carne de ovinos de diferentes categorias quanto aos músculos dos cortes da carcaça (paleta, lombo e perna), utilizaram-se 18 ovinos 1/2 Ile de France $1 / 2$ Ideal (seis cordeiros não-castrados, seis ovelhas de descarte e seis machos adultos castrados). Os animais foram criados em pasto de capim-tifton 85 e receberam suplementação (concentrado). Os cordeiros foram abatidos aos $32 \mathrm{~kg}$, com aproximadamente 5 meses de idade, e as ovelhas e os machos adultos castrados, com $55 \mathrm{~kg}$ e aos 60 meses de idade. Não houve diferenças nos valores de pH 45 minutos e pH 24 horas entre as categorias animais e nos músculos dos cortes da carcaça. A idade de abate influenciou a luminosidade da carne de todos os músculos. O teor de vermelho não diferiu entre os animais adultos, mas foi superior aos valores obtidos na carne dos cordeiros. A carne de animais adultos é mais escura que a de cordeiros. As perdas por cocção no músculo Triceps brachii são maiores que no Longissimus lumborum e Semimembranosus. Os valores de pH e a capacidade de retenção de água são similares entre as categorias animais.
\end{abstract}

Palavras-chave: categorias animais, cor, força de cisalhamento, perdas por cocção, pH

\section{Quality of meats from cuts of lamb and adult sheep carcasses}

ABSTRACT - Eighteen 1/2 Ile de France 1/2 Polwarth sheep (6 no castrated lambs, 6 discarded ewes and 6 discarded wethers) were used to evaluate qualitative traits of meat from different categories of sheep for muscle cuts (shoulder, loin and leg). The animals were raised in grazing system with tifton-85 pastures and supplemented with concentrate. Lambs were slaughtered at $32 \mathrm{~kg}$ body weight, close to 5 months of age. Ewes and wethers were slaughtered with $55 \mathrm{~kg}$ and 60 months of age. There were no differences in $\mathrm{pH}_{45 \mathrm{mim}}$ and $\mathrm{pH}_{24 \mathrm{~h}}$ values among different animal categories and muscles of carcass cuts. There was difference in meat luminosity between lambs and adult animals, considering all the evaluated muscles. The red level did not differ between adult categories, but it was higher than values observed in lambs. In conclusion, meat from adult animals was darker than lamb meat. Considering all animal categories, cooking losses were higher in Triceps brachii compared to Longissimus lumborum and Semimembranosus. Values of $\mathrm{pH}$ and water retention capacity were similar among animal categories.

Key Words: animal categories, color, cooking losses, $\mathrm{pH}$, shear force

\section{Introdução}

O consumo de carne ovina no Brasil é crescente, o que torna necessário produzir em quantidade e qualidade para atender ao consumidor. O mercado consumidor apresenta atualmente elevada exigência quanto à qualidade das características da carne, o que torna necessário o conhecimento dessas características nas diversas faixas de peso dos ovinos destinados ao abate (Bressan et al., 2001). Portanto, é necessário conhecer os parâmetros de qualidade da carne, como pH, cor, capacidade de retenção de água, perdas de água por cocção e maciez, para produzir e processar adequadamente esses produtos, buscando obter alta qualidade da carne e proporcionar maior competitividade entre as demais fontes de origem animal.

A carne pode ser definida como o produto resultante das contínuas transformações que ocorrem no músculo após a morte do animal. Em situações em que se respeitam as condições de bem-estar do animal ante mortem, o pH do músculo após a morte do animal diminui de aproximadamente 7 para 5,5 em decorrência do acúmulo de glicogênio neste período (ante mortem), o que ocasiona a 
transformação em ácido láctico, causada pela ausência de oxigênio nas células, resultando em reações bioquímicas post mortem e gerando a transformação de músculo em carne. O pH é considerado um dos mais importantes parâmetros de qualidade da carne, pois pode interferir nos demais parâmetros (Bonagurio et al., 2003).

A cor da carne é uma característica que o consumidor pode apreciar no momento da compra, determinando, indiretamente, a vida de prateleira. No entanto, a cor da carne é também uma questão cultural, como na Espanha, em que o consumidor prefere carnes de coloração mais claras, enquanto em outros países da Europa, preferem-se carnes de coloração um pouco mais escura (Sañudo et al., 1998).

A maciez da carne é frequentemente o atributo mais importante na satisfação geral do consumidor (Silva Sobrinho et al., 2005) e pode ser definida como a facilidade de mastigar a carne com sensações de penetração, corte e resistência à ruptura.

Sabe-se, também, que a raça, a nutrição, o manejo, o peso de abate e a condição sexual dos ovinos interferem nos parâmetros de qualidade da carne. Todavia, essas avaliações têm sido muito estudadas na categoria representada pelos cordeiros, enquanto as demais categorias, que também têm grande importância econômica e social, estão esquecidas quanto aos aspectos qualitativos da carne. No entanto, é fundamental conhecer os caracteres qualitativos da carne proveniente de ovinos de descarte, pois a ovinocultura está muito avançada tecnicamente, possibilitando a produção de grande número de cordeiras para a reposição das matrizes, o que proporciona o descarte dos animais que não mais produzem com eficiência.

Objetivou-se com este estudo conhecer a qualidade da carne de cordeiros não-castrados e de animais adultos de descarte (ovelhas e machos castrados).

\section{Material e Métodos}

O experimento foi conduzido na Faculdade de Ciências Agrárias e Veterinárias - FCAV/Unesp, localizada em Jaboticabal, São Paulo. Utilizaram-se 18 ovinos Ile de France $\times$ Ideal (seis cordeiros não-castrados, desmamados com aproximadamente $17 \mathrm{~kg}$, seis ovelhas e seis adultos machos castrados, ambos de descarte com peso aproximado de $55 \mathrm{~kg}$ ). Os animais foram everminados e mantidos em pasto de capim-tifton 85 (Cynodon spp), em sistema de lotação intermitente, até o momento do abate.

Os ovinos receberam diariamente suplementação concentrada em quantidade equivalente a $1 \%$ do peso corporal (Tabela 1), fornecida às $17 \mathrm{~h}$ em cochos de madeira que permitiam o acesso simultâneo de todos os animais; um dos cochos foi colocado dentro de um cercado móvel de madeira, de 1,5 m de largura $\times 3,8 \mathrm{~m}$ de comprimento, com ripas de madeira distanciadas $20 \mathrm{~cm}$ uma da outra, no sentido vertical, o que permitiu que apenas os cordeiros tivessem acesso ao concentrado oferecido nesse cocho.

As amostras da pastagem e dos concentrados experimentais foram coletadas para determinação dos teores de matéria seca, proteína bruta, extrato etéreo, cinzas, fibra em detergente neutro e fibra em detergente ácido (Tabela 2), conforme descrito por Silva \& Queiroz (2002).

As amostragens da pastagem foram realizadas pelo método do quadrado, conforme descrito por Gastaldi (1996). Os cordeiros foram abatidos quando atingiram $32 \mathrm{~kg}$ de peso corporal, após jejum de 16 horas de dieta sólida. A insensibilização foi realizada por meio de eletronarcose, quando, então, foram seccionadas as veias jugulares e as artérias carótidas para sangria. Os abates dos cordeiros foram intercalados com os dos ovinos adultos, de modo que, quando um cordeiro foi abatido, realizou-se também o abate de uma ovelha e de um macho adulto de descarte, cujo peso de abate não foi fixado.

Após esfola, evisceração e retirada das extremidades (patas) e da cabeça, as carcaças foram submetidas às medições do pH aos 45 minutos após abate, por meio de um peagômetro digital da marca Jonhis, modelo IPHPJ, introduzindo o eletrodo diretamente nos músculos Triceps brachii da paleta, Semimembranosus da perna e Longissimus lumborum do lombo, efetuando-se três medidas em cada local, no lado direito da carcaça. As carcaças foram, então, transferidas para câmara fria a $4^{\circ} \mathrm{C}$ por 24 horas, quando novamente foi determinado o $\mathrm{pH}$ 24 horas. Ao final desse período, as carcaças foram divididas longitudinalmente em duas meias-carcaças, de modo que a metade direita foi seccionada em cinco regiões anatômicas: paleta, pescoço, costelas, lombo e perna.

Os cortes (paleta, perna e lombo) da meia-carcaça direita foram identificados, armazenados em sacos plásticos e congelados em freezer a $-18^{\circ} \mathrm{C}$, por quatro meses, para facilitar as atividades posteriores das análises de qualidade da carne.

Os cortes foram descongelados em geladeira a $10^{\circ} \mathrm{C}$, por 20 horas, dentro dos sacos plásticos. Após esse período, com auxílio de bisturi e faca, foi separado de cada peça um músculo representativo da patela (Triceps brachii) da perna (Semimembranosus) e do lombo (Longissimus lumborum) para determinação das seguintes características: cor, capacidade de retenção de água, perdas de água por cocção e força de cisalhamento.

Para avaliação da cor, foi utilizado colorímetro Minolta Chrome Meter CR-300, por meio do sistema CIELAB L*, 
Tabela 1 - Composição das dietas experimentais (\% matéria seca)

\begin{tabular}{lcc}
\hline & & Dieta \\
\cline { 2 - 3 } Ingrediente (\%) & Cordeiros & Ovelhas de descarte e machos adultos castrados \\
\hline Grão de milho moído & 54,80 & 60,80 \\
Farelo de trigo & 17,90 & 21,20 \\
Farelo de soja & 24,70 & 15,40 \\
Calcário calcítico & 1,50 & 1,50 \\
Fosfato bicálcico & 0,10 & 0,10 \\
Sal & 0,50 & 0,50 \\
Suplemento mineral ${ }^{1}$ & 0,50 & 0,50 \\
\hline Composição nutricional (\%) & & 86,40 \\
Matéria seca & 87,90 & 4,20 \\
Cinzas & 3,70 & 15,90 \\
Proteína bruta & 20,02 & 3,10 \\
Extrato etéreo & 3,21 & 16,88 \\
Fibra em detergente neutro & 16,70 & 6,73 \\
Fibra em detergente ácido & 5,98 &
\end{tabular}

${ }^{1}$ Suplemento mineral: Zn, 1.600 mg; Cu, 600 mg; Mn, 1.500 mg; Fe, 1.100 mg; Co, 10 mg; I, 27 mg; Na, 62 g; e Se, 22 mg.

Tabela 2 - Composição do capim-tifton 85 (Cynodon spp)

\begin{tabular}{lr}
\hline Forragem & $(\% \mathrm{MS})$ \\
\hline Matéria seca & 24,35 \\
Cinzas & 8,48 \\
Proteína bruta & 9,23 \\
Extrato etéreo & 1,45 \\
Fibra em detergente neutro & 74,82 \\
Fibra em detergente ácido & 41,29 \\
\hline
\end{tabular}

a* e b*, calibrado para um padrão branco em ladrilho. A coloração dos músculos Triceps brachii, Semimembranosus e Longissimus lumborum foi determinada na parte interna desses cortes, 30 minutos após o corte do músculo, para exposição da mioglobina ao oxigênio, de acordo com Cañeque \& Sañudo (2000).

A capacidade de retenção de água foi calculada utilizando-se a metodologia descrita por Hamm, citada por Silva Sobrinho (1999).

Para o cálculo das perdas por cocção, foi utilizado um forno a gás pré-aquecido a $170^{\circ} \mathrm{C}$. Amostras de carnes cruas foram pesadas e colocadas em bandejas com grelhas de ferro e novamente pesadas. Em seguida foram transferidas para o forno, onde foram mantidas a temperatura interna do centro da amostra atingir $75^{\circ} \mathrm{C}$. Em seguida, as bandejas com as grelhas e amostras foram retiradas do forno e, quando esfriaram, foram pesadas novamente para cálculo da porcentagem de perdas por cozimento.

A força de cisalhamento foi determinada utilizando-se as mesmas amostras da análise de perdas de água por cocção, as quais, depois de pesadas, foram cortadas em pedaços de aproximadamente 1,6 × 1,6 × 3,2 cm e, posteriormente, cortadas no sentido transversal das fibras musculares, utilizando-se lâmina Warner-Bratzer acoplada ao aparelho Texture Analyser TA-XT2i, e os valores foram expressos em $\mathrm{kgf} / \mathrm{cm}^{2}$.

O delineamento experimental foi o inteiramente casualizado em esquema fatorial $3 \times 3$ ( 3 categorias animais e 3 cortes da carcaça), e seis repetições utilizando-se análise de variância e comparação de médias pelo teste Tukey a 5\% de probabilidade (SAS, 1996).

\section{Resultados e Discussão}

Não houve interação $(\mathrm{P}>0,05)$ dos valores de $\mathrm{pH}$ para as categorias animais e músculos dos cortes da carcaça (paleta, lombo e perna) com valores médios de $\mathrm{pH} 45$ minutos e pH 24 horas de 6,49 e 5,58, respectivamente. Oliveira et al. (2004), ao avaliarem cordeiros e carneiros da raça Santa Inês, obtiveram pH inicial (60 minutos após abate) de 6,67 e 6,73, respectivamente, e pH final (24 horas após abate) de 5,61 e 5,61, respectivamente, para os músculos Longissimus dorsi e Triceps brachii e não encontraram diferenças entre ovinos jovens ou adultos e o tipo de músculo.

Valores de 5,50 para o $\mathrm{pH} 24$ horas no músculo Longissimus dorsi de ovelhas de descarte $1 / 2$ Ile de France $1 / 2$ Ideal, abatidas aos $50 \mathrm{~kg}$ de peso corporal foram encontrados na literatura (Silva Sobrinho et al., 2004) para a mesma categoria animal utilizada neste estudo, composição genética e músculo (Tabela 3).

Os valores de luminosidade diferiram $(\mathrm{P}<0,01)$ entre as categorias, com maiores valores para o Longissimus lumborum e o Semimembranosus dos cordeiros $(40,42)$ que o Triceps brachii (Tabela 4) e, quanto ao Longissimus lumborum e o Semimembranosus, não ocorreram diferenças 
$(\mathrm{P}>0,05)$. Nos ovinos adultos, houve semelhança $(\mathrm{P}>0,05)$ do valor de luminosidade nos resultados. Macías et al. (1998) avaliaram a cor da carne nos cortes da carcaça de cordeiros castrados e constataram valores para luminosidade de 36,56 , 36,77 e 35,85, respectivamente, para os músculos Biceps femoris, Longissimus dorsi e Semimembranosus.

Resultados de luminosidade similares aos obtidos para os cordeiros (Tabela 4) foram reportados por Russo et al. (1999), que observaram valor de 41,58 no músculo Longissimus lumborum. No músculo Semimembranosus dos animais adultos de descarte, os valores de luminosidade (Tabela 4) foram semelhantes aos encontrados por Silva Sobrinho et al. (2004) no músculo Longissimus dorsi de ovelhas de descarte. Os músculos Triceps brachii, Longissimus lumborum e Semimembranosus não diferiram quanto à luminosidade $(\mathrm{P}>0,05)$ entre os animais adultos, com valores médios de 33,21; 34,51 e 35,47, respectivamente. A categoria animal influenciou $(\mathrm{P}<0,05)$ a luminosidade da carne nos músculos Triceps brachii e Longissimus lumborum dos cordeiros, sendo os valores superiores aos dos animais adultos (Tabela 4). Bressam et al. (2001) observaram que a luminosidade diminuiu de 42,29 para 32,46 com o aumento de 15 para $45 \mathrm{~kg}$ no peso ao abate.

Bonagurio et al. (2003) constataram que a luminosidade da carne diminuiu com o aumento do peso de abate dos ovinos. Silva Sobrinho et al. (2005) reportaram que o valor de luminosidade da carne no músculo Semimembranosus de cordeiros abatidos aos 150 dias foram mais elevados $(38,20)$ que os determinados na carne dos animais abatidos aos 300 dias de idade $(36,80)$, o que indica que a coloração da carne de ovinos se torna mais escura com o aumento da idade e do peso ao abate (Tabela 4).

Esse fato é explicado pelo aumento de mioglobina, que está relacionada à maturidade do animal, pois ovinos adultos apresentam maior quantidade de gordura intramuscular, o que determina menor permeabilidade do capilar e dificulta a transferência de oxigênio entre a fibra muscular. É necessário, portanto, maior quantidade de mioglobina para garantir o aporte de oxigênio adequado (Renerre \& Vilin, 1979, citados por Cañeque \& Sañudo, 2000).

Os resultados de intensidade de vermelho não diferiram $(\mathrm{P}>0,05)$ entre os músculos nos animais adultos, com valor médio de 19,30. Nos cordeiros, a intensidade da cor vermelha foi maior $(\mathrm{P}<0,01)$ no Triceps brachii (Tabela 4) em comparação ao Semimembranosus. O músculo Longissimus lumborum não diferiu $(\mathrm{P}>0,05)$ dos músculos Triceps brachii e Semimembranosus quanto à intensidade da cor vermelha (Tabela 4). Resultados similares foram descritos por Bressan et al. (2001), que verificaram valores similares para intensidade de vermelho nos músculos Longissimus dorsi e Semimembranosus. Os músculos Triceps brachii, Longissimus lumborum e Semimembranosus dos cortes da carcaça dos ovinos adultos não diferiram entre si $(\mathrm{P}>0,05)$ quanto à intensidade da cor vermelha. Sañudo et al. (1996) estudaram a influência do peso de abate e verificaram que a intensidade de vermelho aumentou com o peso de abate. Bressam et al. (2001) constataram valores de intensidade de vermelho de 10,39 e 13,89 com o aumento de $15 \mathrm{~kg}$ para $45 \mathrm{~kg}$ de peso corporal no peso de abate de cordeiros. Dawson et al. (2002) verificaram em ovinos que a intensidade de vermelho aumenta com o peso ao abate, fato confirmado também por Bonagurio et al. (2003).

As médias obtidas para intensidade de amarelo não foram influenciadas $(\mathrm{P}>0,05)$ pelos músculos estudados, com exceção do Triceps brachii, que, para os machos adultos de descarte, foram superiores às das ovelhas e dos cordeiros, que não diferiram entre si $(\mathrm{P}>0,05)$. Os resultados encontrados para a intensidade do amarelo nos músculos Longissimus lumborum e Semimembranosus (Tabela 4)

Tabela 3 - Valores de pH 45 minutos e pH 24 horas nos músculos da paleta (Triceps brachii), do lombo (Longissimus lumborum) e da perna (Semimembranosus) de ovinos de três categorias

\begin{tabular}{lccc}
\hline Cortes da carcaça (músculos) & \multicolumn{2}{c}{ Categoria } & CV\% \\
\cline { 2 - 4 } & Machos adultos castrados & Ovelhas & Cordeiros \\
\hline Paleta (Triceps brachii) & & PH 45 minutos ${ }^{1}$ & 6,51 \\
Lombo (Longissimus lumborum) & 6,49 & 6,42 & 6,53 \\
Perna (Semimembranosus) & 6,48 & 6,47 & 6,55 \\
\hline & 6,49 & 6,48 & 5,50 \\
\hline Paleta (Triceps brachii) & & PH 24 horas $^{2}$ & 5,53 \\
Lombo (Longissimus lumborum) & 5,44 & 5,68 & 5,55 \\
Perna (Semimembranosus) & 5,49 & 5,74 & 3,29 \\
\hline
\end{tabular}

${ }^{1}$ Teste $\mathrm{F}($ músculo $\times$ categoria $)=0,05^{\mathrm{NS}} ;{ }^{2}$ Teste $\mathrm{F}($ músculo $\times$ categoria $)=0,45^{\mathrm{NS}}$. 
Tabela 4 - Luminosidade, intensidade de vermelho e intensidade de amarelo dos músculos da paleta (Triceps brachii), do lombo (Longissimus lumborum) e da perna (Semimembranosus) de ovinos de três categorias

\begin{tabular}{|c|c|c|c|c|}
\hline \multirow[t]{2}{*}{ Corte da carcaça (músculo) } & \multicolumn{3}{|c|}{ Categoria } & \multirow[t]{2}{*}{$\mathrm{CV} \%$} \\
\hline & Machos adultos castrados & Ovelhas & Cordeiros & \\
\hline \multicolumn{5}{|c|}{ Luminosidade $^{1}$} \\
\hline Lombo (Longissimus lumborum) & $34,39 b$ & 34,63b & $40,75 \mathrm{Aa}$ & \multirow[t]{2}{*}{4,92} \\
\hline Perna (Semimembranosus) & $35,20 b$ & $35,74 b$ & $40,10 \mathrm{Aa}$ & \\
\hline Lombo (Longissimus lumborum) & $19,48 \mathrm{a}$ & $19,20 \mathrm{a}$ & $15,39 \mathrm{ABb}$ & \multirow[t]{2}{*}{5,95} \\
\hline \multirow[t]{2}{*}{ Perna (Semimembranosus) } & 19,27 a & $19,18 \mathrm{a}$ & $14,10 \mathrm{Bb}$ & \\
\hline & \multicolumn{3}{|c|}{ Intensidade de amarelo ${ }^{3}$} & \\
\hline Paleta (Triceps brachii) & $6,16 \mathrm{Aa}$ & $4,05 \mathrm{Ab}$ & $2,40 \mathrm{Bb}$ & \multirow[b]{2}{*}{29,62} \\
\hline Lombo (Longissimus lumborum) & $3,29 B$ & $3,51 \mathrm{~A}$ & $5,12 \mathrm{~A}$ & \\
\hline
\end{tabular}

estão de acordo com os reportados por Dawson et al. (2002), que avaliaram a qualidade da carne de ovinos e constataram que a intensidade de amarelo da carne não diferiu entre os animais abatidos aos $36 \mathrm{~kg}$ e $52 \mathrm{~kg}$. Bressam et al. (2001), no entanto, relataram que a intensidade de amarelo da carne aumentou de 6,73 para 8,15 com o aumento de $15 \mathrm{~kg}$ para $45 \mathrm{~kg}$.

A intensidade de amarelo diferiu $(\mathrm{P}<0,01)$ entre os músculos nos machos adultos de descarte: os valores foram maiores no Triceps brachii e menores no músculo Semimembranosus. Para as ovelhas, a intensidade de amarelo não diferiu $(\mathrm{P}>0,05)$ entre os músculos Triceps brachii e Longissimus lumborum, mas diferiu $(\mathrm{P}<0,01)$ da obtida no Semimembranosus. A intensidade de amarelo na carne dos cordeiros não variou $(\mathrm{P}>0,05)$ entre os músculos Triceps brachii e Semimembranosus, mas diferiu $(\mathrm{P}<0,01)$ da obtida no Longissimus lumborum.

Os resultados obtidos para intensidade do amarelo nos músculos Longissimus dorsi e Semimembranosus (Tabela 4) contrariam os encontrados por Macías et al. (1998), que estudaram a cor da carne nos cortes da carcaça de cordeiros e observaram valores similares entre esses músculos. No músculo Longissimus lumborum dos cordeiros, as médias para intensidade do amarelo foram inferiores à determinada por Russo et al. (1999), de 6,53 nesse mesmo músculo. Valor similar ao determinado neste estudo foi relatado por Silva Sobrinho et al. (2004), que encontraram intensidade do amarelo de 3,51 no músculo Longissimus dorsi. A cor da carne é influenciada pela luminosidade e intensidade do vermelho, enquanto a intensidade do amarelo é mais significativa na cor da gordura.

Não houve interação entre categorias animais e músculos dos cortes da carcaça $(\mathrm{P}>0,05)$ para a capacidade de retenção de água da carne, cujos valores médios foram de 56,40\% (Tabela 5), semelhantes aos encontrados de Macías et al. (1998), que não notaram diferença entre os músculos da paleta, lombo e perna. Silva Sobrinho et al. (2004) constataram capacidade de 56,63\% de retenção de água na carne ovina.

As perdas por cocção não foram influenciadas $(\mathrm{P}>0,05)$ pela categoria nem pelo músculo avaliado (Tabela 5), com exceção do Longissimus lumborum, cujos valores foram maiores para os cordeiros em comparação aos ovinos adultos. Lloyd et al. (1981), ao pesquisar ovinos de $54 \mathrm{~kg}$ e 64 kg de peso corporal, não encontraram diferenças nas perdas por cozimento no músculo Longissimus dorsi. Silva Sobrinho et al. (2005) constataram valores similares de perdas de peso ao cozimento no músculo Semimembranosus de cordeiros abatidos aos 150 e 300 dias de idade.

As menores perdas por cozimento nos músculo Longissimus lumborum dos cordeiros (Tabela 5) estão de acordo com o relatado por Bonagurio et al. (2003) de que a perda por cozimento reduz com o aumento do peso ao abate. As perdas por cocção diferiram $(\mathrm{P}<0,01)$ entre as categorias animais e foram maiores no músculo Triceps brachii dos machos castrados, cujo valor foi superior aos dos músculos Longissimus lumborum e Semimembranosus, que não diferiram entre si $(\mathrm{P}>0,05)$. As perdas por cocção do Triceps brachii nas ovelhas foram superiores às dos músculos 
Longissimus lumborum e Semimembranosus, que foram similares entre si $(\mathrm{P}>0,05)$. As perdas por cocção da carne dos cordeiros foram de 67,58\% para o Triceps brachii e de 44,24\% para os músculos Longissimus lumborum e Semimembranosus, que não diferiram entre si $(\mathrm{P}>0,05)$.

As maiores perdas por cocção no músculo Triceps brachii, em comparação àos demais músculos (Tabela 5), pode ser explicada pelo efeito da raça do animal e pelo teor de gordura da carne, uma vez que as perdas por cozimento são menores nas carnes com maior teor de gordura (Sañudo et al., 1997). Perdas por cozimento similares às determinadas nos músculos Longissimus lumborum e Semimembranosus dos ovinos adultos deste estudo foram reportados por Silva Sobrinho (1999), 38,41\%. Bressan et al. (2001), no entanto, em cordeiros de 15 a 45 $\mathrm{kg}$ de peso corporal, encontraram valores mais altos, de $27,2 \%$ e $33,1 \%$, respectivamente, para as perdas ao cozimento dos músculos Longissimus dorsi e Semimembranosus (Tabela 5), o que pode estar relacionado à metodologia adotada na análise. Silva Sobrinho et al. (2004) obtiveram perdas por cozimento de $36,78 \%$ e $36,83 \%$, respectivamente, ao avaliarem os métodos de congelamento lento e rápido do músculo Longissimus dorsi.

A força de cisalhamento foi inflluenciada $(\mathrm{P}<0,01)$ pela categoria animal e pelo músculo avaliado: os maiores valores foram para o Triceps brachii dos machos castrados (Tabela 5), enquanto as ovelhas e os cordeiros não diferiram entre si $\left(2,21 \mathrm{kgf} / \mathrm{cm}^{2}\right)$. Oliveira et al. (2004) também observaram maior força de cisalhamento no músculo Triceps brachii de animais adultos em comparação aos cordeiros.
Ovinos adultos apresentaram maior força de cisalhamento no músculo Semimembranosus em relação aos cordeiros (Tabela 5). Gularte et al. (2000) avaliaram a maciez da carne de animais de diversos sexos e pesos de abate e observaram que a força de cisalhamento no músculo Longissimus dorsi de ovinos abatidos aos 9 meses de idade $\left(3,43 \mathrm{kgf} / \mathrm{cm}^{2}\right)$ foi maior que nos animais abatidos com 7 e 8 meses $\left(2,08 \mathrm{kgf} / \mathrm{cm}^{2}\right.$ e $2,31 \mathrm{kgf} / \mathrm{cm}^{2}$, respectivamente). Silva Sobrinho et al. (2005) também observaram maior força de cisalhamento na carne de animais abatidos aos 300 dias de idade em comparação aos abatidos com 150 dias. Esses resultados podem ser explicados pelo fato de ovinos mais velhos apresentarem maior diâmetro das fibras musculares e maior número de ligações cruzadas dessas fibras, que resultam em carne mais dura. A força de cisalhamento no Longissimus lumborum não diferiu $(\mathrm{P}>0,05)$ entre as categorias, com média de $1,65 \mathrm{kgf} / \mathrm{cm}^{2}$ (Tabela 5), fato relatado também por Kemp et al. (1981), que não observaram diferenças na força de cisalhamento do músculo Longissimus dorsi de ovinos abatidos com 30 a $60 \mathrm{~kg}$ de peso corporal, fato relacionado à maior quantidade de gordura intramuscular no músculo Longissimus dorsi de ovinos mais velhos, que resulta em carne mais macia.

A força de cisalhamento diferiu $(\mathrm{P}<0,01)$ entre todos os músculos avaliados. Nos machos castrados, os maiores valores foram determinados no músculo Triceps brachii e os menores no Longissimus lumborum. Nas ovelhas, não diferiu $(\mathrm{P}>0,05)$ entre os músculos Triceps brachii e Semimembranosus e o valor obtido foi superior ao determinado no Longissimus lumborum. Nos cordeiros, a força de cisalhamento não diferiu $(\mathrm{P}>0,05)$ entre os músculos Triceps

Tabela 5 - Capacidade de retenção de água, perdas por cocção e força de cisalhamento dos músculos da paleta (Triceps brachii), do lombo (Longissimus lumborum) e da perna (Semimembranosus) de ovinos de três categorias

\begin{tabular}{|c|c|c|c|c|}
\hline \multirow[t]{2}{*}{ Cortes da carcaça (músculos) } & \multicolumn{3}{|c|}{ Categoria } & \multirow[t]{2}{*}{ CV\% } \\
\hline & Machos adultos castrados & Ovelhas & Cordeiros & \\
\hline \multicolumn{5}{|c|}{ Capacidade de retenção de água $(\%)^{1}$} \\
\hline Paleta (Triceps brachii) & 55,35 & 57,62 & 56,11 & \multirow{3}{*}{6,05} \\
\hline Lombo (Longissimus lumborum) & 56,43 & 57,51 & 58,03 & \\
\hline Perna (Semimembranosus) & 53,72 & 54,46 & 57,47 & \\
\hline Paleta (Triceps brachii) & $66,47 \mathrm{~A}$ & 66,73A & $67,58 \mathrm{~A}$ & \multirow{3}{*}{7,00} \\
\hline Lombo (Longissimus lumborum) & 39,33Bb & $38,32 \mathrm{Bb}$ & $46,44 \mathrm{Ba}$ & \\
\hline \multirow[t]{2}{*}{ Perna (Semimembranosus) } & $39,08 \mathrm{~B}$ & $37,31 \mathrm{~B}$ & $42,05 B$ & \\
\hline & \multicolumn{3}{|c|}{ Força de cisalhamento $\left(\mathrm{kgf} / \mathrm{cm}^{2}\right)^{3}$} & \\
\hline Paleta (Triceps brachii) & $3,84 \mathrm{Aa}$ & $2,43 \mathrm{Ab}$ & $2,00 \mathrm{Ab}$ & 24,40 \\
\hline
\end{tabular}


brachii e Longissimus lumborum, cujo valor (1,90 kgf/ $\left.\mathrm{cm}^{2}\right)$ foi superior ao determinado no Semimembranosus. Esses valores determinados nos machos castrados e nas ovelhas (Tabela 5) foram similares aos encontrados por Silva Sobrinho et al. (2004), de 2,82 kgf/cm². Oliveira et al. (2004) também estudaram a carne de ovinos adultos e jovens e constataram que o músculo Longissimus dorsi é mais macio que o Triceps brachii, provavelmente em decorrência da maior atividade física dos músculos da paleta em relação aos do lombo do animal.

\section{Conclusões}

$\mathrm{O}$ pH dos músculos Triceps brachii, Longissimus lumborum e Semimembranosus e a capacidade de retenção de água da carne não são influenciados pela idade e pelo sexo e peso de abate. A carne proveniente de ovinos adultos é mais escura que a dos animais jovens. As perdas de água por cocção no músculo Triceps brachii são maiores que no Longissimus lumborum e Semimembranosus, independentemente da categoria ovina. A maciez da carne do músculo Longissimus lumborum é similar entre as categorias animais estudadas, mas difere entre os músculos Triceps brachii e Semimembranosus. Os músculos dos cortes da carcaça e as categorias ovinas não determinam diferenças expressivas na qualidade da carne que justifiquem a indicação ao consumidor de qual produto a ser adquirido; prevalece, portanto, a escolha do próprio consumidor e a relação custo-benefício.

\section{Agradecimentos}

Ao Conselho Nacional de Pesquisa e Desenvolvimento (CNPq), pela bolsa concedida durante o curso de mestrado.

\section{Literatura Citada}

BONAGURIO, S.; PÉREZ, J.R.O.; GARCIA, I.F.F. et al. Qualidade da carne de cordeiros Santa Inês puros e mestiços com Texel abatidos com diferentes pesos. Revista Brasileira de Zootecnia, v.32, n.6, p.1981-1991, 2003.

BRESSAN, C.; PRADO, O.V.; PÉREZ, J.R.O. et al. Efeito do peso ao abate de cordeiros Santa Inês e Bergamácia sobre as características físico-químicas da carne. Ciência e Tecnologia de Alimentos, v.21, n.3, p.293-303, 2001.

CAÑEQUE, V.; SAÑUDO, C. Metodología para el estúdio de la calidad de la canal y de la carne en rumiantes. Madrid:
Instituto Nacional de Investigación y Tecnología y Alimentaria, 2000. 255p.

DAWSON, L.E.R.; CARSON, A.F.; MOSS, B.W. Effects of crossbred ewe genotype and ram genotype on lamb meat quality from the lowland sheep flock. Journal of Animal Science, v.13, n.9, p.195-204, 2002.

GASTALDI, K.A. Taxas de lotação influenciando a produção ovina. 1996. 118f. Trabalho de conclusão de curso (Graduação em Zootecnia) - Universidade Estadual Paulista/Faculdade de Ciências Agrárias e Veterinárias, Jaboticabal, 1996.

GULARTE, M.A.; TREPTOW, R.O.; POUEY, J.L.F. et al. Idade e sexo na maciez da carne ovina da raça Corriedale. Ciência Rural, v.30, n.3, p.485-488, 2000.

KEMP, J.D.; MAHYUDDIN, M.; ELY, D.G. et al. Effect of feeding systems, slaughter weight and sex on organoleptic properties, and fatty acid composition of lamb. Journal of Animal Science, v.51, n.2, p.321-330, 1981.

LLOYD, W.R.; SLYTER, A.L.; COSTELLO, W.J. Effect of breed, sex, and final weight on feedlot perfomance, carcass characteristcs and meat palatability of lambs. Journal of Animal Science, v.51, n.2, p.316-320, 1981.

MACÍAS, J.A.; GONZÁLEZ, F.A.N.; ALMEIDA, F.A.R. et al. Calidad de la canal y de la carne de borregos Pelibuey castrados. Revista Técnica Pecuária do México, v.36, n.3, p.225-232, 1998.

OLIVEIRA, I.; SILVA, T.J.P.; FREITAS, M.Q. et al. Caracterização do processo de rigor mortis em músculos de cordeiros e carneiros da raça Santa Inês e maciez da carne. Revista Acta Scientiae Veterinariae, v.32, n.1, p.25-31, 2004.

RUSSO, C.; PREZIUSO, G.; CASAROSA, L. et al. Effect of diet energy source on the chemical-physical characteristics of meat and depot fat of lambs carcasses. Small Ruminant Research, v.33, n.1, p.77-85, 1999.

SAÑUDO, C.; SANTOLARIA, M.P., MARIA, G. et al. Influence of carcass weight on instrumental and sensory lamb meat quality in intensive production systems. Meat Science, v.42, n.2, p.195-202, 1996.

SAÑUDO, C.; CAMPO, M.M., SIERRA, I. et al. Breed effect on carcase and meat quality of suckling lambs. Meat Science, v.46, n.4, p.357-365, 1997.

SAÑUDO, C.; NUTE, G.R.; CAMPO, M.M. et al. Assessment of comercial lamb meat quality by british and spanish taste panels. Meat Science, v.48, n.1/2, p.91-100, 1998.

SILVA SOBRINHO, AG. Body composition and characteristics of carcass from lambs of different genotypes and ages at slaughter. 1999. 54f. Dissertation (PostDoctorate in Sheep Meat Production) - Massey University, Palmerston North, 1999.

SILVA SOBRINHO, A.G.; ZEOLA, N.M.B.; SOUZA, P.A. et al. Qualidade da carne ovina in natura e congelada por diferentes métodos. In: REUNIÃO ANUAL DA SOCIEDADE BRASILEIRA DE ZOOTECNIA, 41., 2004, Campo Grande. Anais... Campo Grande: Sociedade Brasileira de Zootecnia, [2004]. (CD-ROM).

SILVA SOBRINHO, A.G.; PURCHAS, R.W.; KADIM, I.T. et al. Características de qualidade da carne de ovinos de diferentes genótipos e idades ao abate. Revista Brasileira de Zootecnia, v.34, n.3, p.1070-1078, 2005.

SILVA, D.J.; QUEIROZ, A.C. Análise de alimentos: métodos químicos e biológicos. 5.ed. Viçosa, MG: Imprensa Universitária, 2002. 235p.

STATISTICAL ANALYSIS SYSTEM - SAS. User's guide to statistics. Version 6.12. Cary: North Caroline State University, 1996. (CD-ROM). 